\title{
An Outbreak of a Respiratory Disorder at a Russian Swine Farm Associated with the Co-Circulation of PRRSV1 and PRRSV2
}

\author{
Sergei Raev ${ }^{1, *}$, Anton Yuzhakov ${ }^{1}{ }^{1}$, Alexandr Bulgakov ${ }^{1}\left({ }^{1}\right.$, Ludmila Kostina ${ }^{1}$, \\ Alexei Gerasianinov ${ }^{2}$, Oleg Verkhovsky ${ }^{3}$, Alexei Zaberezhny ${ }^{1}$ and Taras Aliper ${ }^{1}$ \\ 1 Federal State Budget Scientific Institution “Federal Scientific Center VIEV”, 109428 Moscow, Russia; \\ anton_oskol@mail.ru (A.Y.); bulgakov_ad@mail.ru (A.B.); lvkostina@mail.ru (L.K.); \\ zaberezhny@mail.ru (A.Z.); coronavirus@yandex.ru (T.A.) \\ 2 Siberian Agrarian Group, 634009 Tomsk, Russia; gerasyanovav@sagro.ru \\ 3 Laboratory of Virology, Diagnostics and Prevention Research Institute for Human and Animal Diseases, \\ 123098 Moscow, Russia; info@dpri.ru \\ * Correspondence: raevsergey33@gmail.com; Tel.: +1-330-601-4796
}

Received: 20 September 2020; Accepted: 11 October 2020; Published: 15 October 2020

\begin{abstract}
We conducted a cross-sectional study to identify the major respiratory pathogen responsible for an outbreak of respiratory disease at a swine farm in West Siberia in 2019. We discovered that the peak of morbidity and mortality coincided with a high level of porcine reproductive and respiratory syndrome virus (PRRSV) 1 and 2-related viremia. Based on longer PRRSV2 viremia, the dominant role of PRRSV2 over PRRSV1 in the outbreak was assumed. Phylogenetic analysis revealed that the PRRSV1 strain belonged to sub-genotype 2-one of the predominant groups of genotype 1 PRRSVs in Russia. A partial open reading frame 7 sequence of the PRRSV2 isolate demonstrated a high identity with modified live vaccine-related strains from Denmark (93\%) and wild-type VR2332 (92\%). We identified the first instance of PRRSV1/PRRSV2 mixed infection in Russia. This finding indicates that further field investigations are needed to access PRRSV2 epidemiology in eastern Europe.
\end{abstract}

Keywords: PRRSV1; PRRSV2; PCV2; ORF7; cross-sectional; phylogenetic analysis; antibody detection

\section{Introduction}

Porcine reproductive and respiratory syndrome (PRRS) has been simultaneously described in the USA and Europe and remains a major problem for the pig industry. Economic losses associated with this disease are estimated at US\$664 million per year globally. In sows, reproductive failure is characterized by abortions, fetus mummification, stillbirths, and birth of weak offspring. Growing piglets mainly demonstrate respiratory symptoms such as dyspnea, coughing, and fever [1].

Further investigations have revealed that the causative agent of this disease is porcine reproductive and respiratory syndrome virus (PRRSV), for which two genotypes have been described: the European (PRRSV1) and North American (PRRSV2) genotypes. Significant genetic (50-60\% identity) and antigenic differences eventually led to their divergence into two separate species: Betaarterivirus suid 1 (the European type, or PRRSV1) and Betaarterivirus suid 2 (the North American type, or PRRSV2) [2]. Both viruses belong to genus Betaarterivirus in the Variartevirinae subfamily and Arteriviridae family. PRRSV's genome comprises about 15,000 nucleotides and contains ten open reading frames (ORFs) including ORF7, which encodes the nucleocapsid protein [3].

Shortly after PRRSV's discovery, both PRRSV1 and PRRSV2 were isolated beyond their original places of detection, namely Europe and North America, respectively. PRRSV1 strains are currently present in Europe, North America, and Asia, and PRRSV2 strains are predominant in North America 
and Asia but commonly found in Europe. Since PRRSV2 was first isolated in Denmark in 1995; similar (vaccine-related) strains were detected in Europe on numerous occasions. Despite wild-type PRRSV2 strains being isolated in Germany and Hungary, modified live vaccine (MLV)-related strains remain a major group of genotype 2 PRRS viruses circulating in Europe [4-9].

Co-circulation of PRRSV1 and PRRSV2 within one continent and the absence of cross-protection between them have logically resulted in dual infections at farms. For instance, both PRRSV1 and PRRSV2 were found at $24.2 \%$ of infected farms in South Korea [10].

In Russia, PRRS is mainly caused by PRRSV1-1 (including the so-called atypical Russian group of viruses) and PRRSV1-2 [9,11,12]. Only limited data are available on PRRSV2 epidemiology in Russia. One PRRS outbreak caused by highly pathogenic PRRSV2 (JXA1-related) was described in 2007 [13]. Notably, PRRSV2 is periodically detected on its own or simultaneously with PRRSV1 by veterinary diagnostic laboratories in Russia. However, due to the comparably low incidence of PRRSV2 detection, it is not considered and often goes unnoticed.

In August 2019, an outbreak of acute respiratory disease was recorded at a swine farm in Kemerovo region, Russia. The outbreak was mainly characterized by dispone, fever, and anorexia. According to the farm's veterinary department data, morbidity and mortality (up to 40\%) peaked in 7- to 12-week-old piglets. This 1500-sow farrow-to-finish farm was located more than $100 \mathrm{~km}$ away from another swine unit. The sow vaccination schedule included quarterly immunization with a killed vaccine against PRRSV (Lelystad-based) porcine parvovirus, pseudorabies virus, and leptospirosis. Piglets received a porcine circovirus type 2 (PCV2) subunit vaccine and a killed vaccine (one dose each) and two doses of live classical swine fever vaccine. Based on the latest laboratory update at this farm in 2012, the farm was endemic for PRRSV, PCV2, and Mycoplasma hyopneumoniae (M.hyo). Considering the current outbreak and the long period of time that had passed since the last laboratory investigations were carried out, a decision was made to conduct a cross-sectional surveillance study.

The main goal of this investigation was to identify the major causative agent of respiratory outbreak at the farm and partially characterize the genome sequences of the isolated viruses.

\section{Materials and Methods}

\subsection{Samples}

The blood samples were taken from piglets ages $0,3,7,12,16,20$, and 26 weeks ( 8 to 10 animals per age group). Serum samples were collected on the same day and stored frozen at $-70{ }^{\circ} \mathrm{C}$ before analysis.

\subsection{ELISA}

All serum samples were tested for the presence of antibodies against PRRSV capsid protein using two commercial ELISA test kits. The first kit, INgezim PRRS Universal (Ingenasa, Madrid Spain), is prepared on the basis of both PRRSV1 and PRRSV2 recombinant nucleoproteins. This kit detects antibodies against both PRRSV1 and PRRSV2 designated as "pan-PRRSV antibodies". The second assay, RRSS-SEROTEST (Vetbiochem, Moscow, Russia), is capable of detecting antibodies against PRRSV1 only. Both ELISA test kits were used in accordance with the manufacturers' instructions.

\subsection{PCR and Sequencing}

PRRSV , PCV2, swine influenza virus (SIV), porcine parvovirus virus (PPV), and porcine respiratory coronavirus $(P R C V)$ in serum samples were detected using commercial polymerase chain reaction (PCR) kits (Vetbiochem, Russia) according to the manufacturer's instructions. In all serum samples, detection was performed using commercial real-time polymerase chain reaction (PCR) kits (Vetbiochem, Russia) in accordance with the manufacturer's instructions. According to previous data, both ORF5 and ORF7 sequences might be chosen for PRRSV1 classification [8]. We used the following primers for the amplification and sequencing of PRRSV1 ORF7, kindly provided by Ivan Trus (Ghe University, Ghent, Belgium): 5'-TGGCCCCTGCCCAICACGT-3' (PRRSV1-ORF7-F) and 
5'-TGATCGCCCTAATTGAATAGGTGACT-3' (PRRSV1-ORF7-R). For the amplification and sequencing of PRRSV2 ORF7, we used two pairs of nested primers. For the first round of PCR, we used the following primers: 5'-TTCTGGCCCCTGCCCAYC-3' (F7) and 5'-CGCCCTAATTGAATAGGTGAC-3' (R7). For the second round of PCR as well as for sequencing, we used $5^{\prime}$-CCAAATAACAACGGCAAG-3' (AmerF7_12) and 5'-TCATGCTGAGGGTGATG-3' (AmerR7_12). The expected size of PCR products of PRRSV1 ORF7 and PRRSV2 ORF7 was around 644 and 368 nucleotides, respectively. A one-tube, real-time RT-PCR kit (Alpha Ferment, Moscow, Russia) was used to perform reverse-transcription polymerase chain reaction (RT-PCR). The PCR amplification program consisted of $10 \mathrm{~min}$ at $50{ }^{\circ} \mathrm{C}$, 5 min at $95^{\circ} \mathrm{C}, 35$ cycles at $95^{\circ} \mathrm{C}$ for $15 \mathrm{~s}, 55^{\circ} \mathrm{C}$ for $15 \mathrm{~s}$, and $72{ }^{\circ} \mathrm{C}$ for $30 \mathrm{~s}$, as well as 1 cycle of $5 \mathrm{~min}$ at $72{ }^{\circ} \mathrm{C}$. The amplification products were visualized in $1 \%$ agarose gel in a buffer containing a mixture of Tris base, acetic acid and EDTA (x1 TAE) after which purification was performed using Monarch DNA Gel Extraction Kit (New England Biolabs, MA, USA) in accordance with the manufacturer's instructions. Sanger sequencing was performed using the Big DyeßTerminator v.3.1 Cycle Sequencing Kit (Applied Biosystems, CA, USA) in accordance with the manufacturer's instructions, and the primers specified above. The nucleotide sequences of the genome fragments were determined using an AB3130 genomic automated analyzer (Applied Biosystems, USA). The nucleotide sequences were analyzed using Lasergene 11.1.0. (DNASTAR, WI, USA). Multiple alignment was performed using MUSCLE (MEGA 7.0.18). Phylogenetic dendrograms were plotted using the maximum likelihood method, GTR model (MEGA 7.0.18). The topology of the trees was confirmed following 1000 bootstrapping replication steps.

\section{Results}

\subsection{Viremial ELISA}

Viral RNA was not detected in sera from either newborn or 3-week-old piglets, whereas in 7-week-old piglets, PRRSV1 and PRRSV2, and viral RNA were present in $50 \%$ and $40 \%$ of serum samples, respectively (Figure 1). The mixed RNA belonging to both viruses was detected in only one 7-week-old piglet. In sharp contrast to PRRSV1, where the RNA was not present in samples from 12-week-old animals, PRRSV2 viremia was detected in $60 \%$ of 12 -week-old piglets. Simultaneously, PCV2 DNA was detected in $20 \%$ of 12 -week-old piglets, and viremia rates increased with age until the age of 20 weeks, with $40 \%$ of the piglets tested being viremic. All serum samples were negative for SIV, PPV , and PRCV.

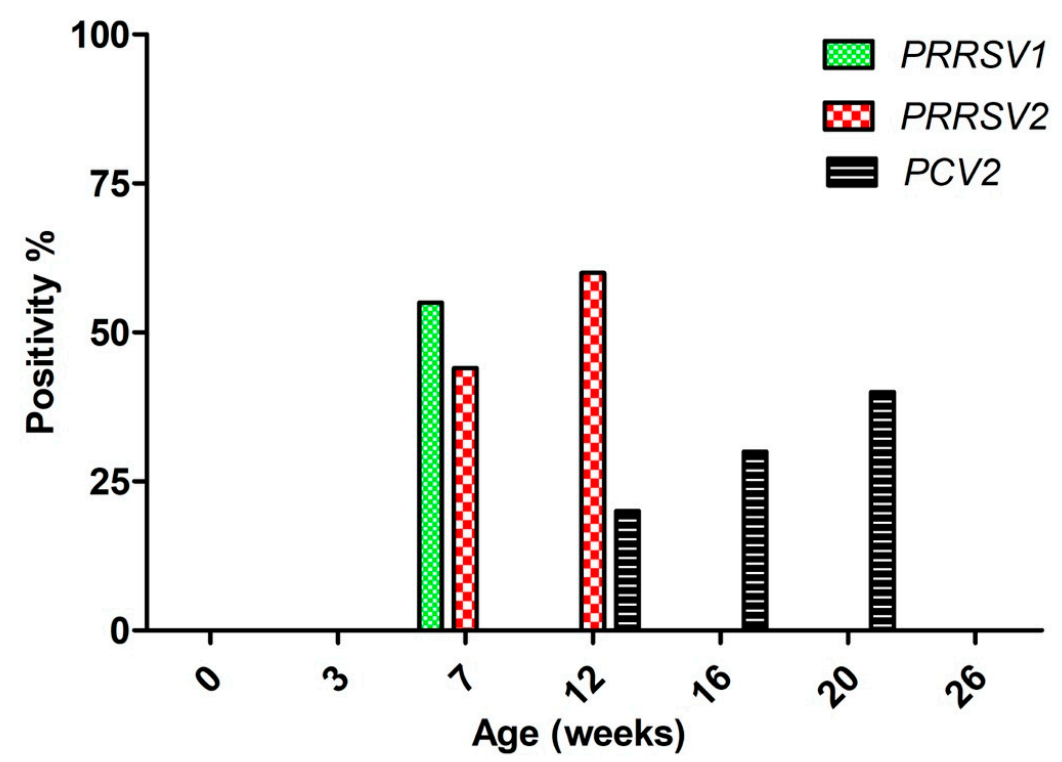

Figure 1. Viremia evaluation of PRRSV1, PRRSV2, and PCV2 in different age groups. 
Whereas the total concentration of PRRSV1-specific antibodies showed a clear tendency to rise from 3 weeks (when all samples were seronegative) to 20 weeks of age, a minor numerical decrease in the total level of antibodies against both strains from 3 to 7 weeks of age was detected (Figure 2).

Comprehensive analysis of total PRRSV antibodies (to both genotypes) and PRRSV1-specific antibodies (Table 1) did not reveal any certain tendency. PRRSV2 RNA was found in an only numerically higher (three out of nine) proportion of PRRSV1 IgG negative/total PRRSV Ig-positive samples compared to PRRSV1 (one out of nine).

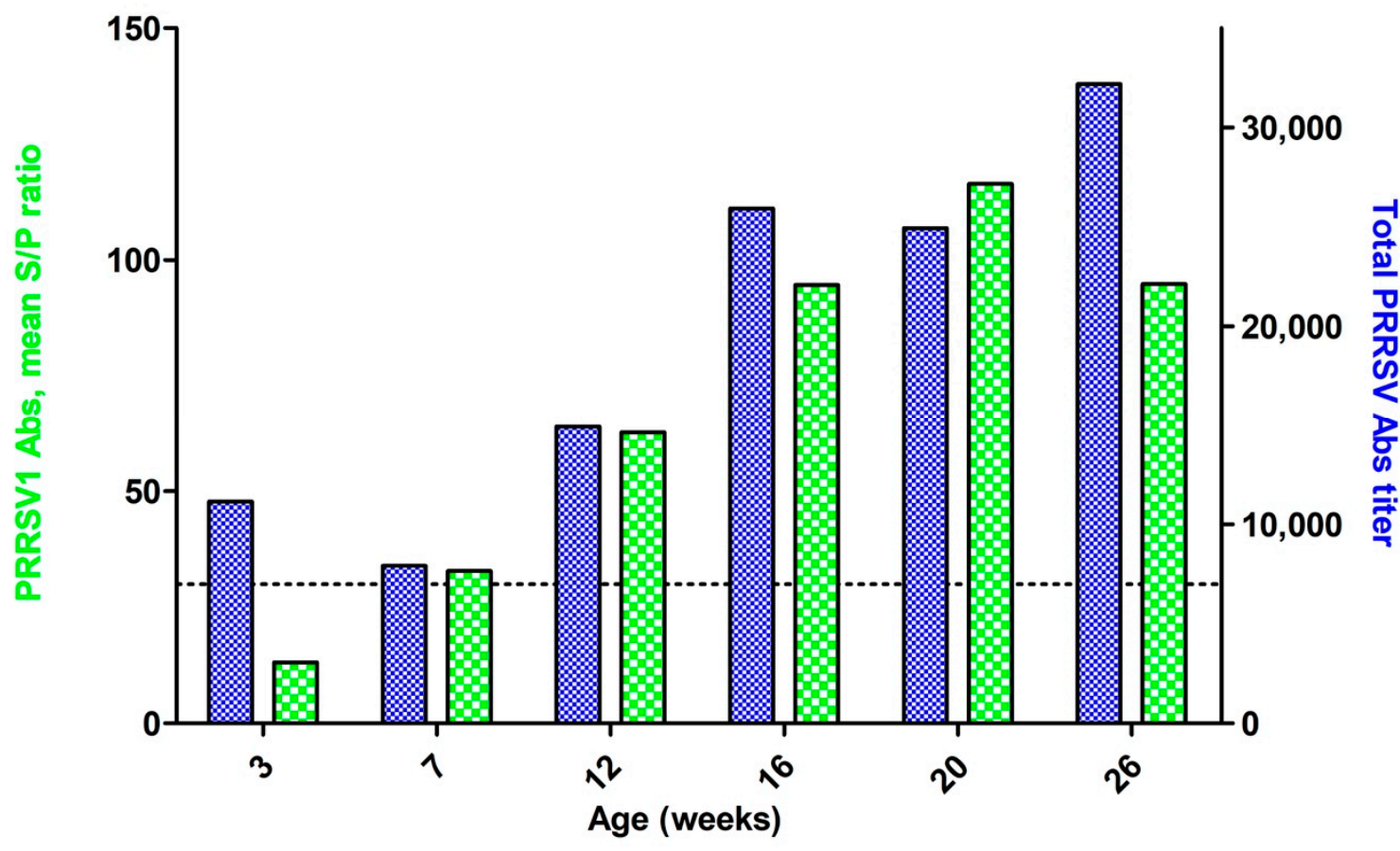

Figure 2. Anti-PRRSV1 (green) and pan-PRRSV (blue) antibody detection.

Table 1. Antibody detection. Detection of antibodies to PRRSV1 (positive samples highlighted in green), antibodies to both, PRRSV1 and PRRSV2 (positive samples highlighted in blue), RNA from PRRSV1 (positive samples highlighted in green), and RNA from PRRSV2 (positive samples highlighted in red).

\begin{tabular}{|c|c|c|c|c|c|}
\hline \multirow{2}{*}{ Week } & \multirow{2}{*}{ Animal \# } & \multicolumn{2}{|c|}{ Antibody Detection (ELISA) } & \multicolumn{2}{|c|}{ Viremia Detection (PCR) } \\
\hline & & PRRSV1 & Pan-PRRSV & PRRSV1 & PRRSV2 \\
\hline \multirow{8}{*}{3} & 1 & & & & \\
\hline & 2 & & & & \\
\hline & 3 & & & & \\
\hline & 4 & & & & \\
\hline & 5 & & & & \\
\hline & 6 & & & & \\
\hline & 7 & & & & \\
\hline & 8 & & & & \\
\hline \multirow{9}{*}{7} & 1 & & & & \\
\hline & 2 & & & & \\
\hline & 3 & & & & \\
\hline & 4 & & & & \\
\hline & 5 & & & & \\
\hline & 6 & & & & \\
\hline & 7 & & & & \\
\hline & 8 & & & & \\
\hline & 9 & & & & \\
\hline
\end{tabular}


Table 1. Cont.

\begin{tabular}{|c|c|c|c|c|c|}
\hline \multirow{2}{*}{ Week } & \multirow{2}{*}{ Animal \# } & \multicolumn{2}{|c|}{ Antibody Detection (ELISA) } & \multicolumn{2}{|c|}{ Viremia Detection (PCR) } \\
\hline & & PRRSV1 & Pan-PRRSV & PRRSV1 & PRRSV2 \\
\hline \multirow{10}{*}{12} & 1 & & & & \\
\hline & 2 & & & & \\
\hline & 3 & & & & \\
\hline & 4 & & & & \\
\hline & 5 & & & & \\
\hline & 6 & & & & \\
\hline & 7 & & & & \\
\hline & 8 & & & & \\
\hline & 9 & & & & \\
\hline & 10 & & & & \\
\hline \multirow{10}{*}{16} & 1 & & & & \\
\hline & 2 & & & & \\
\hline & 3 & & & & \\
\hline & 4 & & & & \\
\hline & 5 & & & & \\
\hline & 6 & & & & \\
\hline & 7 & & & & \\
\hline & 8 & & & & \\
\hline & 9 & & & & \\
\hline & 10 & & & & \\
\hline \multirow{10}{*}{20} & 1 & & & & \\
\hline & 2 & & & & \\
\hline & 3 & & & & \\
\hline & 4 & & & & \\
\hline & 5 & & & & \\
\hline & 6 & & & & \\
\hline & 7 & & & & \\
\hline & 8 & & & & \\
\hline & 9 & & & & \\
\hline & 10 & & & & \\
\hline \multirow{8}{*}{26} & 1 & & & & \\
\hline & 2 & & & & \\
\hline & 3 & & & & \\
\hline & 4 & & & & \\
\hline & 5 & & & & \\
\hline & 6 & & & & \\
\hline & 7 & & & & \\
\hline & 8 & & & & \\
\hline
\end{tabular}

\subsection{Sequencing Results}

Serum samples from 7-week-old piglets were used for PRRSV1 (sample \#4) and for PRRSV2 (sample \#6) sequencing. The ORF7 sequences were deposited into the GenBank sequence database under the accession numbers ORF7_Kem19EU-MT344126 and ORF7_Kem19NA-MT344127. The ORF7_Kem19EU-MT344126 (PRRSV1) has 378 nucleotides, and further phylogenetic analysis revealed that this isolate belongs to subtype 2 of PRRSV1 (Figure 3). This strain is most closely related to strains Eig иAus from Lithuania and strain Gk from the European part of Russia [8].

The partial nucleotide sequence of ORF7_Kem19NA-MT344127 (PRRSV2) comprises 337 nucleotides (Figure 4). The comparative analysis of partial sequence ORF7_Kem19NA-MT344127 against those of reference strains demonstrated a high nucleotide identity with wild-type VR2332 (92\%) and MLV-related strains from Denmark: KC577602.1_DK-2004-3-1 and AF095479.1_strain_17704A (93\%) [14]. The level of identity between the Hungarian non-MLV-related strain KM514315.1:14861-15232_Hungary_102_2012 and ORF7_Kem19NA-MT344127 was only 81\%. Nucleotide identity with GU256774.1, the only one PRRSV2 strain detected in Russia, was $88 \%$. 


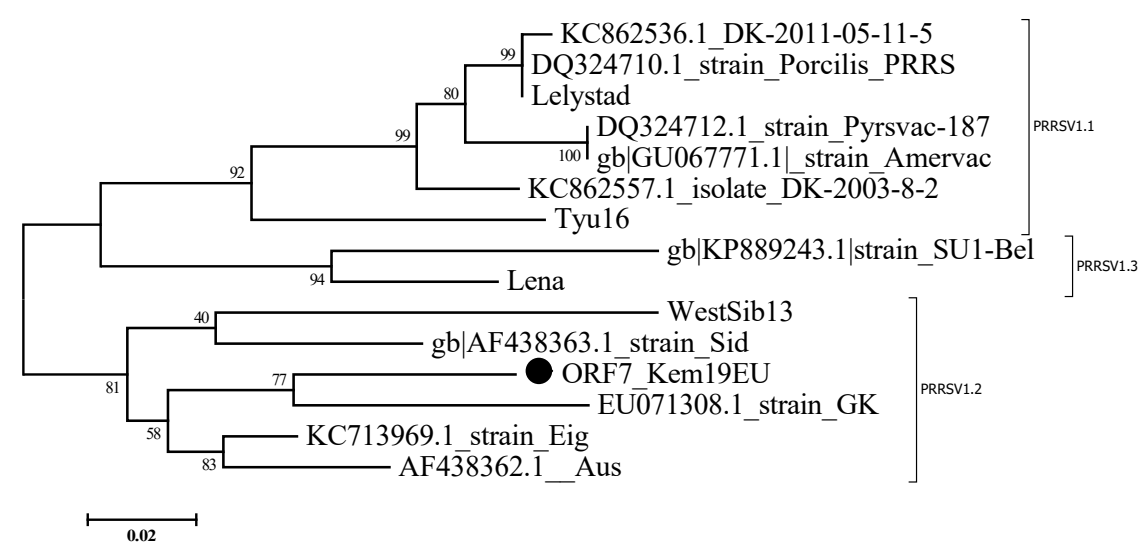

Figure 3. Phylogenetic trees of PRRSV1 strains based on complete ORF7 nucleotide sequences. Bootstrap confidence limits are presented at each node. The Kem19EU strain is designated by a circle $(\bullet)$. Strains are designated in the following format: GenBank accession number_name.

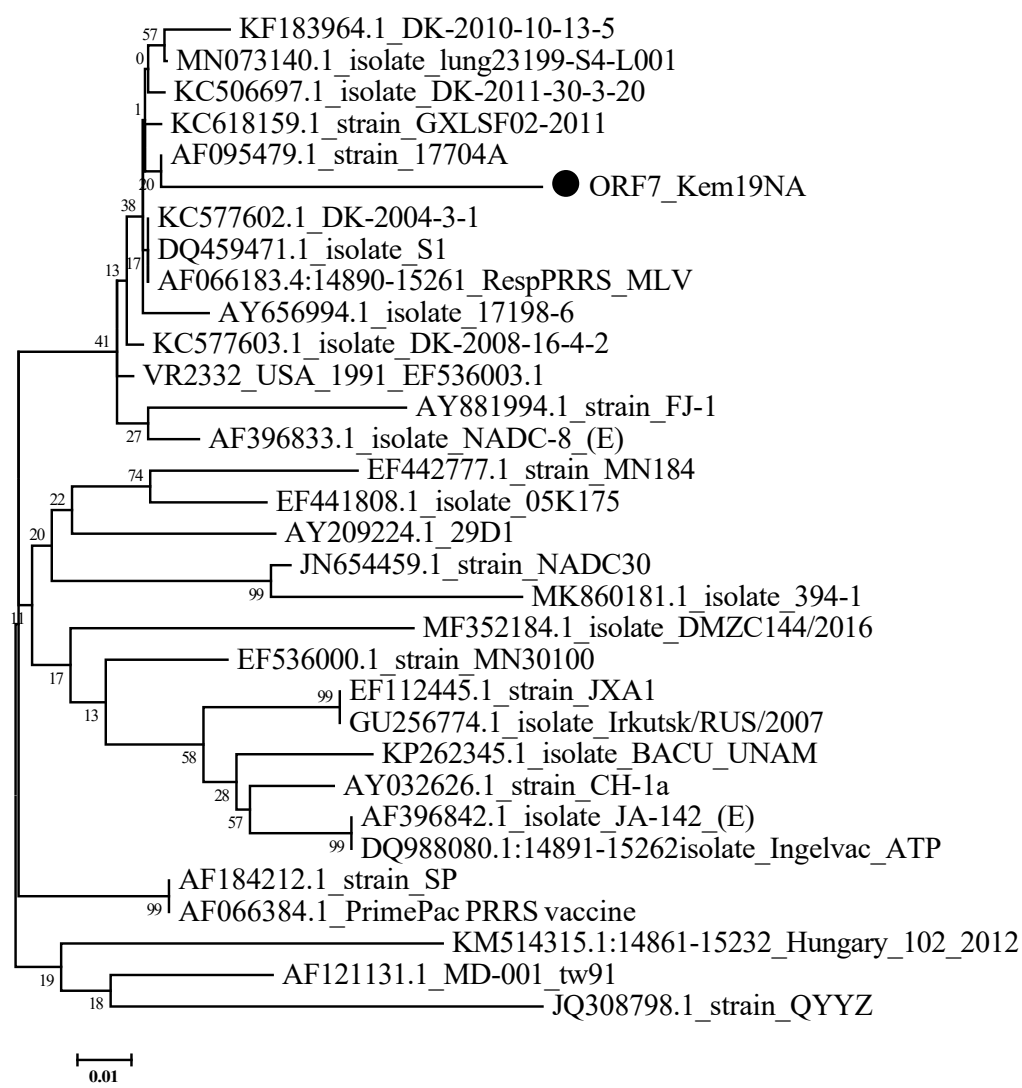

Figure 4. Phylogenetic trees of PRRSV2 strains based on partial ORF7 nucleotide sequences. Bootstrap confidence limits are presented at each node. The Kem19NA strain is designated by a circle $(\bullet)$. Strains are designated in the following format: GenBank accession number_name.

Further genetic analysis (Figure 5a) revealed that Kem19NA had an amino acid insertion of lysine (K) at position 47 in the nuclear localization signal (NLS) sequence of the N protein. Similar amino acid insertions are present in PRRSV1-2 strains (Figure 5b), which is remarkable given the debate on the evolution of PRRSV1 and PRRSV2. 


\section{a}

KC506697_isolate_DK-2011-30-3KC618159_strain_GXLSF02-2011 AF184212

EF536000_strain_MN30100

EF442777_strain_MN184 KC577603_isolate_DK-2008-16-4 KC577602_DK-2004-3-1 KR183964_DK-2010-10-13-5 AY881994-MD-001_tw9 AY032626_strain_CH-1a AF095479_strain $17704 \mathrm{~A}$ KM514315_isolate_Hungary_102_2 EF112445_strain_JXA1 GU256774_isolate_Irkutsk/RUS/2 JQ308798_strain_DQ459471_train_NADC3 AF396833 isolate NADC- 8 MN073140 isolate lung $23199-S$ AF396842 isolate JA-142 (E) AY656994_isolate_17198-6 MK860181_isolate_394-1

A 066183 strain_RespPRRS_MLV DQ98808_ isolate Ingelvac AT KP262345 isolate BACU UNAM AY209224_isolate_29D1 EF441808 isolate $05 \mathrm{~K} 175$ MF352184_isolate_DMZC144/201 MT344127_isolate_Kem19NA

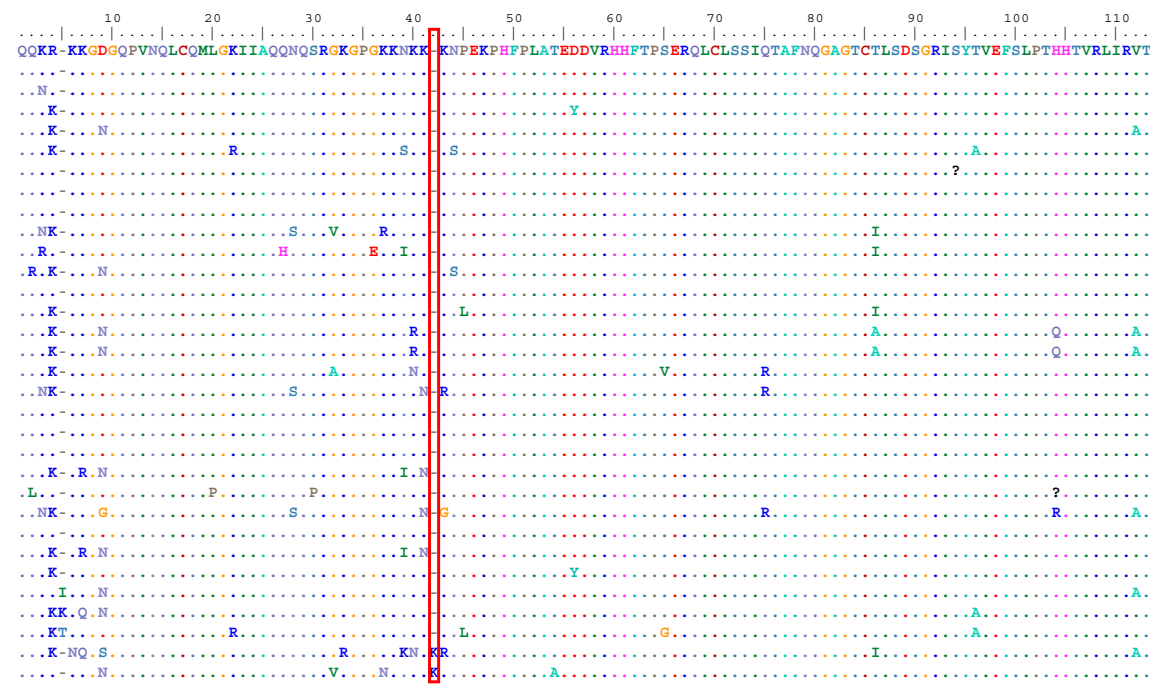

b

M96262_strain_Lelystad GU067771_strain_Amervac KC862557_isolate_DK-2003-8-2

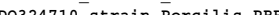
QQ324710_strain_Porcilis_PRF DQ324712_strain_PYrsvacJF802085 strain_Len KP889243 strain SU1-Be strain_Bor 59 * strain_ILI-6* KC668221_isolate Westsib1 AF438363_strain_sid AF438362_strain_Aus KC713969_strain_Eig EU071308_strain_GK

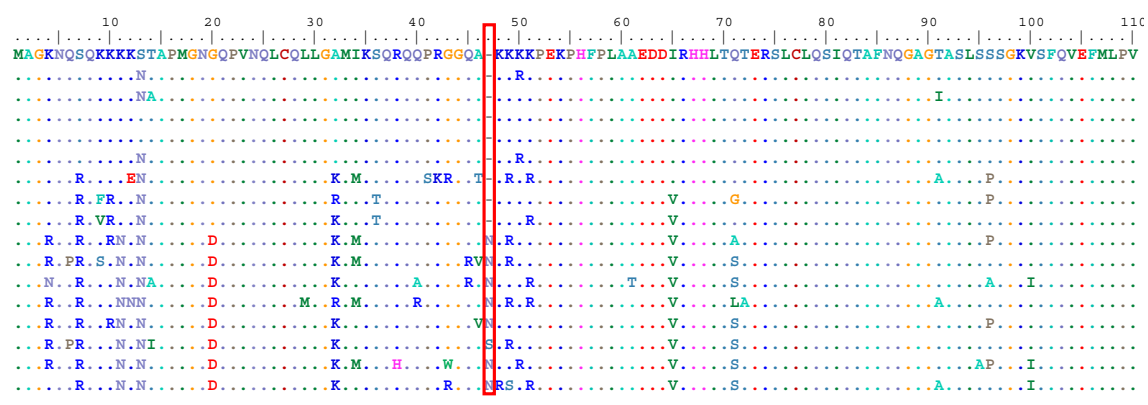

M96262_strain_Lelystad

GU067771_strain_Amervac

KC862557_isolate_DK-2003-8-2

KC862536_isolate_DK-2011-05-11

DQ24710_strain_Porcilis_PR

DQ324712_strain_Pyrsvac-187

JF802085 strain Lena

KP889243_strain_SU1-Be

strain_Bor59*
strain_ILI-6*

KC668221_isolate Westsib1

AF438363_strain_Sid

AF438362_strain_Au

KC713969_strain_Eig

EU071308_strain_GR

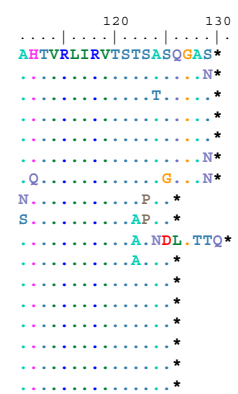

Figure 5. Partial alignment of amino acid sequences derived from ORF7 of PRRSV2 (a) and complete alignment of amino acid sequences derived from ORF7 of PRRSV1 (b) strains. Dots and hyphens represent identical amino acid positions and gapped positions, respectively. The red box denotes insertions in the investigated strains. The sequences of the strains indicated by an asterisk $(*)$ were kindly provided by Prof. Dr. Tomasz Stadejek (Warsaw University of Life Sciences, Poland) and Dr. Sara Botti (Parco Tecnologico Padano, Italy).

\section{Discussion}

The results of this study indicate that the respiratory disease outbreak correlated with increased PRRSV-related viremia rates, while it did not correlate with the prevalence of another major respiratory pathogen, PCV2. Remarkably, PRRSV2-related viremia peaked in a more pronounced manner and lasted longer compared to PRRSV1-related viremia. This might be an indication of the predominant role of PRRSV2 in respiratory outbreak development.

We assume that whereas total PRRSV antibodies from piglets aged 12 weeks might be considered as post-infectious, at 3 weeks of age, these antibodies are of maternal origin. Due to differences in sensitivity and specificity between the test kits used in this study and simultaneous PRRSV1 and 
PRRSV2 viremia detection, our effort to distinguish between PRRSV1 and PRRSV2 antibodies by using universal (PRRSV1/PRRSV2) and PRRSV1-specific ELISA test kits cannot describe the real situation. After the initial introduction of PRRSV2 into Europe, differentiation between antibodies against PRRSV of different genotypes became crucial to PRRSV diagnosis. An ELISA assay for simultaneous detection and differentiation between antibodies to PRRSV1 and PRRSV2 was developed [15], but no such ELISA test kit is commercially available in Russia. Hence, the use of universal PCR test kits for simultaneous detection of both viruses is the only option to prevent PRRSV2 from remaining undetected.

Data on the comparative pathogenicity of PRRSV1, PRRSV2, and mixed infection are scarce. In one study, the predominant role of PRRSV2 over PRRSV1 in the course of PRRSV1-1/PRRSV2 (lineage 1) mixed infection was demonstrated on the basis of replication and pathogenicity levels [16]. Significant differences in the pathogenicity of different genotypes/lineages of PRRSV1 and PRRSV2 should be considered in the case of mixed infection. Further experimental studies will be required to establish the impact of at least PRRSV1-2 and PRRSV2 (lineage 5) presence. Despite recombination never being demonstrated between PRRSV1 and PRRSV2 strains, the simultaneous presence of PRRSV1 and PRRSV2 might also be considered a possible risk for the emergence of recombinant strains. The use of MLV vaccines under such circumstances may only increase this risk $[17,18]$.

The idea that PRRSV1 is a homogenous group of viruses was revised after the discovery of genetically distinct isolates from eastern Europe, especially from Belarus and Russia [9]. Thus, the detection of a new member of PRRSV1-2 in the present study offers additional proof of the wide distribution of this genotype in Russia. Isolates of PRRSV1-1 (including the so-called Russian group of viruses), PRRSV1-2, and PRRSV1-3 differ significantly in pathogenicity $[11,12,19,20]$. Marked differences in the nucleotide sequence between PRRSV1-2 isolates and PRRSV1 vaccine strains, which all belong to PRRSV1-1, including differences in the ORF5 sequence (which encodes a major epitope: the envelope glycoprotein), could potentially hamper vaccine efficacy [12]. The benefits of using currently available vaccines against PRRSV1-2, be they live, inactivated, or subunit, remain unknown. The circulation of PRRSV2 further complicates the search for working control strategies, as no vaccines against PRRSV2 are currently available in Russia.

Phylogenetic analysis of a partial ORF7 sequence of the PRRSV2 strain isolated in the course of this study revealed that this strain was very dissimilar ( $88 \%$ identity) to the only known PRRSV2 strain (JXA1-related, sublineage 8.7) detected in East Siberia, Russia, in 2007. This suggests that a different instance of PRRSV2 introduction into Russia occurred at a certain point in addition to the one resulting in the outbreak of 2007. Further genetic analysis (including an ORF5 sequence) should be performed to classify this isolate more precisely.

High homology within lineage 5 of PRRSV2 makes the distinction between the Ingelvac PRRS MLV vaccine strain, its parental strain $V R$-2332, VR-2332-related wild-type isolates, and vaccine-derived isolates very complicated and requires at least ORF5 sequence analysis [18]. The Boehringer MLV vaccine has not been licensed in Russia. However, there are known cases of the detection of an MLV-like PRRSV1 strain in Vietnam, regardless of the corresponding type of vaccine not being licensed in that country [21]. Notably, one locally-produced MLV vaccine against PRRSV2 has been licensed but is currently commercially unavailable in Russia, and the sequence of the vaccine virus has not yet been published. It is also conceivable that the virus originated from breeder animals infected with an MLV-related PRRSV2 strain. It is well known that MLV-related strains (including strains that have a high identity level with ORF7_Kem19NA-MT344127) are widely spread in Denmark, one of the major exporters of breeding pigs [14]. For example, the average number of pigs imported from Denmark to Russia has been more than 3000 animals a year over the past three years. Finally, an independent introduction of a wild-type $V R 2332$-related strain could result in the emergence of the ORF7_Kem19NA-MT344127 virus.

In the course of infection, NLS interacts with nuclear transport proteins, leading to the penetration of the $\mathrm{N}$ protein into the nucleus and nucleolus of infected cells [22]. A critical role of this element of the $\mathrm{N}$ protein in pathogenesis has been shown [23]. A similar insertion is known to be found 
in a Mexican field isolate DMZC144/2016 (GenBank ID MF352184.1) [24]. This might indicate that both Kem19NA and DMZC144/2016 have a nucleoprotein consisting of 124 amino acids in length. Complete ORF7 sequencing should be performed to confirm this assumption. Interestingly, Kem19NA and DMZC144/2016 are genetically distant from each other, their nucleotide identity being only $87 \%$, suggesting that this insertion may not be lineage-specific.

\section{Conclusions}

To the best of our knowledge, this is the first report of PRRSV1/PRRSV2 co-circulation in Russia. Considered alongside data from field veterinary laboratories, our observations indicate that PRRSV2 circulates in both the European and Asian parts of the country. The prevalence and distribution of PRRSV2 in Russia therefore warrants further investigation.

Author Contributions: S.R., A.Y., and A.G. conceptualized the study; A.Y., L.K., A.G., A.B., and S.R. conducted the experiments and analysis; S.R. and A.Y. prepared the original draft of the manuscript; the manuscript was co-written, reviewed and edited by O.V., A.Z. and T.A.; The research was supervised by T.A.; A.Y. and S.R. were in charge of project administration; A.Y. applied for funding. All authors have read and agreed to the published version of the manuscript.

Funding: The work was carried out at the Federal State Budget Scientific Institution Federal Scientific Center VIEV and funded by the Russian Science Foundation (grant no. 19-16-00102).

Acknowledgments: The authors would like to thank Alexander Mishin and Alexander Skrylev for their administrative support and Yana Streltsova for her excellent technical assistance. We thank Tomasz Stadejek and Sara Botti for providing the sequences of the strains Bor-59 and ILI-6.

Conflicts of Interest: The authors declare no conflict of interest.

\section{References}

1. Done, S.H.; Paton, D.J. Porcine reproductive and respiratory syndrome: Clinical disease, pathology and immunosuppression. Vet. Rec. 1995, 136, 32-35. [CrossRef]

2. International Committee on Taxonomy of Viruses (ICTV)Release EC 50, Washington, DC, July 2018 Email Ratification February 2019 (MSL \#34). Available online: https://talk.ictvonline.org/taxonomy/p/taxonomyhistory?taxnode_id=201851832 (accessed on 16 April 2020).

3. Snijder, E.J.; Meulenberg, J.J. The molecular biology of arteriviruses. J. Gen. Virol. 1998, 79, 961-979. [CrossRef]

4. Balka, G.; Hornyák, Á.; Bálint, Á.; Kiss, I.; Kecskeméti, S.; Bakonyi, T.; Rusvai, M. Genetic diversity of porcine reproductive and respiratory syndrome virus strains circulating in Hungarian swine herds. Vet. Microbiol. 2008, 127, 128-135. [CrossRef]

5. Dewey, C.; Charbonneau, G.; Carman, S.; Hamel, A.; Nayar, G.; Friendship, R.; Eernisse, K.; Swenson, S. Lelystad-like strain of porcine reproductive and respiratory syndrome virus (PRRSV) identified in Canadian swine. Can. Vet. J. La Rev. Veter- Can. 2000, 41, 493-494.

6. Ropp, S.L.; Wees, C.E.M.; Fang, Y.; Nelson, E.A.; Rossow, K.D.; Bien, M.; Arndt, B.; Preszler, S.; Steen, P.; Christopher-Hennings, J.; et al. Characterization of Emerging European-Like Porcine Reproductive and Respiratory Syndrome Virus Isolates in the United States. J. Virol. 2004, 78, 3684-3703. [CrossRef] [PubMed]

7. Shi, M.; Lam, T.T.-Y.; Hon, C.-C.; Murtaugh, M.P.; Davies, P.R.; Hui, R.K.-H.; Li, J.; Wong, L.T.-W.; Yip, C.-W.; Jiang, J.-W.; et al. Phylogeny-Based Evolutionary, Demographical, and Geographical Dissection of North American Type 2 Porcine Reproductive and Respiratory Syndrome Viruses. J. Virol. 2010, 84, 8700-8711. [CrossRef]

8. Stadejek, T.; Oleksiewicz, M.B.; Scherbakov, A.V.; Timina, A.M.; Krabbe, J.S.; Chabros, K.; Potapchuk, D. Definition of subtypes in the European genotype of porcine reproductive and respiratory syndrome virus: Nucleocapsid characteristics and geographical distribution in Europe. Arch. Virol. 2008, 153, 1479-1488. [CrossRef]

9. Stadejek, T.; Stankevicius, A.; Murtaugh, M.P.; Oleksiewicz, M.B. Molecular evolution of PRRSV in Europe: Current state of play. Vet. Microbiol. 2013, 165, 21-28. [CrossRef] 
10. Kang, H.; Yu, J.E.; Shin, J.-E.; Kang, A.; Kim, W.-I.; Lee, C.; Lee, J.; Cho, I.-S.; Choe, S.-E.; Cha, S.-H. Geographic distribution and molecular analysis of porcine reproductive and respiratory syndrome viruses circulating in swine farms in the Republic of Korea between 2013 and 2016. BMC Vet. Res. 2018, 14, 160. [CrossRef]

11. Yuzhakov, A.G.; Raev, S.A.; Shchetinin, A.M.; Gushchin, V.A.; Alekseev, K.P.; Stafford, V.V.; Komina, A.K.; Zaberezhny, A.D.; Gulyukin, A.M.; Aliper, T.I. Full-genome analysis and pathogenicity of a genetically distinct Russian PRRSV-1 Tyu16 strain. Vet. Microbiol. 2020, 247, 108784. [CrossRef]

12. Yuzhakov, A.G.; Raev, S.A.; Skrylev, A.N.; Mishin, A.M.; Grebennikova, T.V.; Verkhovsky, O.A.; Zaberezhny, A.D.; Trus, I.; Nauwynck, H.J.; Aliper, T.I. Genetic and pathogenic characterization of a Russian subtype 2 PRRSV-1 isolate. Vet. Microbiol. 2017, 211, 22-28. [CrossRef] [PubMed]

13. Kukushkin, S.A.; Baybikov, T.Z.; Scherbakov, A.V.; Timina, A.M.; Baborenko, E.P.; Puzankova, O.P.; Pronin, I.A.; Fomin, A.E. First Outbreak of Atypical Porcine Reproductive and Respiratory Syndrome in Russia Caused by Highly Pathogenic Chinese-Like Prrs Virus. NA PRRSV Symp. 2008, 2008, 23-26.

14. Storgaard, T.; Oleksiewicz, M.; Bøtner, A. Examination of the selective pressures on a live PRRS vaccine virus. Arch. Virol. 1999, 144, 2389-2401. [CrossRef]

15. Sørensen, K. Blocking ELISA's for the distinction between antibodies against European and American strains of porcine reproductive and respiratory syndrome virus. Vet. Microbiol. 1998, 60, 169-177. [CrossRef]

16. Choi, K.; Lee, J.; Park, C.; Jeong, J.; Chae, C. Comparison of the Pathogenesis of Single or Dual Infections with Type 1 and Type 2 Porcine Reproductive and Respiratory Syndrome Virus. J. Comp. Pathol. 2015, 152, 317-324. [CrossRef]

17. Bøtner, A.; Strandbygaard, B.; Sorensen, K.J.; Have, P.; Madsen, K.G.; Madsen, E.S.; Alexandersen, S. Appearance of acute PRRS-like symptoms in sow herds after vaccination with a modified live PRRS vaccine. Vet. Rec. 1997, 141, 497-499. [CrossRef]

18. Opriessnig, T.; Halbur, P.G.; Yoon, K.-J.; Pogranichniy, R.M.; Harmon, K.M.; Evans, R.; Key, K.F.; Pallares, F.J.; Thomas, P.; Meng, X.J. Comparison of Molecular and Biological Characteristics of a Modified Live Porcine Reproductive and Respiratory Syndrome Virus (PRRSV) Vaccine (Ingelvac PRRS MLV), the Parent Strain of the Vaccine (ATCC VR2332), ATCC VR2385, and Two Recent Field Isolates of PRRSV. J. Virol. 2002, 76, 11837-11844. [CrossRef] [PubMed]

19. Karniychuk, U.; Geldhof, M.; Vanhee, M.; Doorsselaere, J.V.; A Saveleva, T.; Nauwynck, H.J. Pathogenesis and antigenic characterization of a new East European subtype 3 porcine reproductive and respiratory syndrome virus isolate. BMC Vet. Res. 2010, 6, 30. [CrossRef] [PubMed]

20. Stadejek, T.; Larsen, L.E.; Podgórska, K.; Bøtner, A.; Botti, S.; Dolka, I.; Fabisiak, M.; Heegaard, P.M.; Hjulsager, C.K.; Huc, T.; et al. Pathogenicity of three genetically diverse strains of PRRSV Type 1 in specific pathogen free pigs. Vet. Microbiol. 2017, 209, 13-19. [CrossRef]

21. Nguyen, N.T.; Nguyen, T.T.-D.; Nguyen, T.T.; Le, H.T.-T.; Nguyen, S.G.; Vo, H.K.; Do, K.V.-A. Genetic Analysis of ORF5 Porcine Reproductive and Respiratory Syndrome Virus (PRRSV) Isolated in Vietnam. Microbiol. Immunol. 2013, 57, 518-526. [CrossRef]

22. Rowland, R.; Kervin, R.; Kuckleburg, C.; Sperlich, A.; Benfield, D.A. The localization of porcine reproductive and respiratory syndrome virus nucleocapsid protein to the nucleolus of infected cells and identification of a potential nucleolar localization signal sequence. Virus Res. 1999, 64, 1-12. [CrossRef]

23. Lee, C.; Hodgins, D.; Calvert, J.G.; Welch, S.-K.W.; Jolie, R.; Yoo, D. Mutations within the nuclear localization signal of the porcine reproductive and respiratory syndrome virus nucleocapsid protein attenuate virus replication. Virology 2006, 346, 238-250. [CrossRef] [PubMed]

24. Martínez-Bautista, N.R.; Sciutto-Conde, E.; Cervantes-Torres, J.; Segura-Velazquez, R.; García, M.C.M.; Ramírez-Mendoza, H.; Ortega, M.E.T.; Alvarez, J.D.; Castillo-Juárez, H.; Sánchez-Betancourt, J.I. Phylogenetic analysis of ORF5 and ORF7 of porcine reproductive and respiratory syndrome (PRRS) virus and the frequency of wild-type PRRS virus in México. Transbound. Emerg. Dis. 2018, 65, 993-1008. [CrossRef]

Publisher's Note: MDPI stays neutral with regard to jurisdictional claims in published maps and institutional affiliations. 